\title{
Light clusters production as a probe to nuclear symmetry energy
}

\author{
Lie-Wen Chen, ${ }^{1, *}$ C. M. Ko, ${ }^{1}$ and Bao-An $\mathrm{Li}^{2}$ \\ ${ }^{1}$ Cyclotron Institute and Physics Department, Texas A\&M University, College Station, Texas 77843-3366, USA \\ ${ }^{2}$ Department of Chemistry and Physics, P.O. Box 419, Arkansas State University, State University, Arkansas 72467-0419, USA
}

(Received 6 March 2003; published 14 July 2003)

\begin{abstract}
Using an isospin-dependent transport model for heavy-ion collisions induced by neutron-rich nuclei at intermediate energies, we study the production of light clusters such as deuteron, triton, and ${ }^{3} \mathrm{He}$ via coalescence of nucleons. We find that both the yields and the energy spectra of these light clusters are affected significantly by the density dependence of nuclear symmetry energy, with a stiffer symmetry energy giving a larger yield.

DOI: 10.1103/PhysRevC.68.017601

PACS number(s): 25.70.Pq, 21.30.Fe, 21.65.+f, 24.10.Lx
\end{abstract}

The energy per particle of an asymmetric nuclear matter with density $\rho$ and an isospin asymmetry $\delta=\left(\rho_{n}-\rho_{p}\right) / \rho$, where $\rho_{n}$ and $\rho_{p}$ are, respectively, its neutron and proton densities, is usually approximated by a parabolic law [1], i.e.,

$$
E(\rho, \delta)=E(\rho, 0)+E_{\mathrm{sym}}(\rho) \delta^{2},
$$

where $E(\rho, 0)$ is the energy per particle of symmetric nuclear matter and $E_{\text {sym }}(\rho)$ is the nuclear symmetry energy. Although the nuclear symmetry energy at normal nuclear matter density $\rho_{0}=0.16 \mathrm{fm}^{-3}$ has been determined to be around $30 \mathrm{MeV}$ from the empirical liquid-drop mass formula [2,3], its values at other densities are poorly known. Studies based on various theoretical models also give widely different predictions [4]. Lack of this knowledge has hampered our understanding of both the structure of radioactive nuclei [5-8] and many important issues in nuclear astrophysics [9-11], such as the nucleosynthesis during presupernova evolution of massive stars and the properties of neutron stars $[9,11]$. However, recent advance in radioactive nuclear beam facilities provides the opportunity to study the density dependence of the nuclear symmetry energy. Theoretical studies have already shown that in heavy-ion collisions induced by neutronrich nuclei, the effect of nuclear symmetry energy can be studied via the preequilibrium neutron/proton ratio [12], the isospin fractionation [13-16], the isoscaling in multifragmentation [17], the proton differential elliptic flow [18], the neutron-proton differential transverse flow [19], the $\pi^{-}$to $\pi^{+}$ratio [20], and two-nucleon correlation functions [21].

In this work, we study the production of deuteron, triton, and ${ }^{3} \mathrm{He}$ in heavy-ion collisions induced by neutron-rich nuclei by means of the coalescence model based on the nucleon phase space distribution functions from an isospin-dependent transport model. It is found that both the multiplicities and energy spectra of these light clusters are sensitive to the density dependence of nuclear symmetry energy, but not to the isospin-independent part of nuclear equation of state and the in-medium nucleon-nucleon cross sections. Therefore, light clusters production in heavy-ion collisions induced by

\footnotetext{
*On leave from Department of Physics, Shanghai Jiao Tong University, Shanghai 200030, China.
}

neutron-rich nuclei provides another possible method for extracting useful information about the nuclear symmetry energy.

The coalescence model has been used extensively in describing the production of light clusters in heavy-ion collisions at both intermediate [22-25] and high energies [26,27]. In this model, the probability for producing a cluster is determined by its Wigner phase-space density and the nucleon phase-space distribution at freeze out. Explicitly, the multiplicity of a $M$-nucleon cluster in a heavy ion collision is given by [26]

$$
\begin{aligned}
N_{M}= & G \int d \mathbf{r}_{i_{1}} d \mathbf{q}_{i_{1}} \cdots d \mathbf{r}_{i_{M-1}} d \mathbf{q}_{i_{M-1}} \\
& \times\left\langle\sum_{i_{1}>i_{2}>\cdots>i_{M}} \rho_{i}^{W}\left(\mathbf{r}_{i_{1}}, \mathbf{q}_{i_{1}} \cdots \mathbf{r}_{i_{M-1}}, \mathbf{q}_{i_{M-1}}\right)\right\rangle .
\end{aligned}
$$

In the above, $\mathbf{r}_{i_{1}}, \ldots, \mathbf{r}_{i_{M-1}}$ and $\mathbf{q}_{i_{1}}, \ldots, \mathbf{q}_{i_{M-1}}$ are, respectively, the $M-1$ relative coordinates and momenta taken at equal time in the $M$-nucleon rest frame; $\rho_{i}^{W}$ is the Wigner phase-space density of the $M$-nucleon cluster; and $\langle\cdots\rangle$ denotes event averaging. The spin-isospin statistical factor for the cluster is given by $G$, and its value is $3 / 8$ for deuteron and $1 / 3$ for triton or ${ }^{3} \mathrm{He}$, with the latter including the possibility of coalescence of a deuteron with another nucleon to form a triton or ${ }^{3} \mathrm{He}$ [28].

For the deuteron Wigner function, it is obtained from the Hulthén wave function, i.e.,

$$
\phi=\sqrt{\frac{\alpha \beta(\alpha+\beta)}{2 \pi(\alpha-\beta)^{2}}} \frac{e^{-\alpha r}-e^{-\beta r}}{r}
$$

with parameters $\alpha=0.23 \mathrm{fm}^{-1}$ and $\beta=1.61 \mathrm{fm}^{-1}$ to reproduce the measured deuteron root-mean-square radius of 1.96 fm [29]. As in Ref. [26], we express the Hulthén wave function in terms of 15 arbitrary Gaussian functions and determine their strengths and ranges by a least square fit. For triton and ${ }^{3} \mathrm{He}$, their Wigner functions are obtained from the product of three ground-state wave functions of a spherical harmonic oscillator with its parameters adjusted to reproduce the measured root-mean-square radii of triton and ${ }^{3} \mathrm{He}$, i.e., 
$1.61 \mathrm{fm}$ and $1.74 \mathrm{fm}$, respectively [30]. Normal Jacobian coordinates for a three-particle system are then introduced to derive the Wigner functions for triton and ${ }^{3} \mathrm{He}$ as in Ref. [26].

The space-time distribution of nucleons at freeze out is obtained from an isospin-dependent Boltzmann-UehlingUhlenbeck (IBUU) transport model (e.g., Refs. $[12,19,20,31])$. For a review of the IBUU model, we refer the reader to Ref. [1]. The IBUU model includes explicitly the isospin degree of freedom through different proton and neutron initial distributions as well as their different mean-field potentials and two-body collisions in subsequent dynamic evolutions. For the nucleon-nucleon cross sections, we use as default the experimental values in free space. In order to study the effects due to the isospin dependence of in-medium nucleon-nucleon cross sections $\sigma_{\text {medium }}$, we also use a parametrization obtained from the Dirac-Brueckner approach based on the Bonn A potential [32]. For experimental freespace cross sections, the neutron-proton cross section is about a factor of 3 larger than the neutron-neutron or protonproton cross sections. On the other hand, the in-medium nucleon-nucleon cross sections used here have smaller magnitudes and weaker isospin dependence than $\sigma_{\exp }$ but strong density dependence. For the isoscalar potential, we use as default the Skyrme potential with an incompressibility $K_{0}$ $=380 \mathrm{MeV}$. This potential has been shown to reproduce the transverse flow data from heavy ion collisions equally well as a momentum-dependent soft potential with $K_{0}$ $=210 \mathrm{MeV}[33,34]$.

The IBUU model is solved with the test particle method [35]. Although the mean-field potential is evaluated with test particles, only collisions among nucleons in each event are allowed. Light cluster production from coalescence of nucleons is treated similar as nucleon-nucleon collisions, i.e., only nucleons in the same event are allowed to coalesce to light clusters. Results presented in the following are obtained with 20000 events using 100 test particles for a physical nucleon.

For the density dependence of symmetry energy, we adopt the parametrization used in Ref. [36] for studying the properties of neutron stars, i.e.,

$$
E_{\text {sym }}(\rho)=E_{\text {sym }}\left(\rho_{0}\right) u^{\gamma},
$$

where $u \equiv \rho / \rho_{0}$ is the reduced density and $E_{\text {sym }}\left(\rho_{0}\right)$ $=30 \mathrm{MeV}$ is the symmetry energy at normal nuclear matter density. In the following, we consider the two cases of $\gamma$ $=0.5$ (soft) and $\gamma=2$ (stiff) to explore the large range of $E_{\text {sym }}(\rho)$ predicted by many-body theories [10].

We consider the reaction of ${ }^{52} \mathrm{Ca}+{ }^{48} \mathrm{Ca}$, which has an isospin asymmetry $\delta=0.2$ and can be studied future at Rare Isotope Accelerator Facility. In the present study, nucleons are considered as being frozen out when their local densities are less than $\rho_{0} / 8$ and subsequent interactions do not cause their recapture into regions of higher density. Shown in Figs. $1(\mathrm{a}-\mathrm{c})$ are time evolutions of the average multiplicity of deuteron, triton, and ${ }^{3} \mathrm{He}$ from central collisions of ${ }^{52} \mathrm{Ca}+{ }^{48} \mathrm{Ca}$ at $E=80 \mathrm{MeV} /$ nucleon by using the soft (solid curve) and stiff (dashed curve) symmetry energies. It is seen that production of these light clusters from a neutron-rich reaction

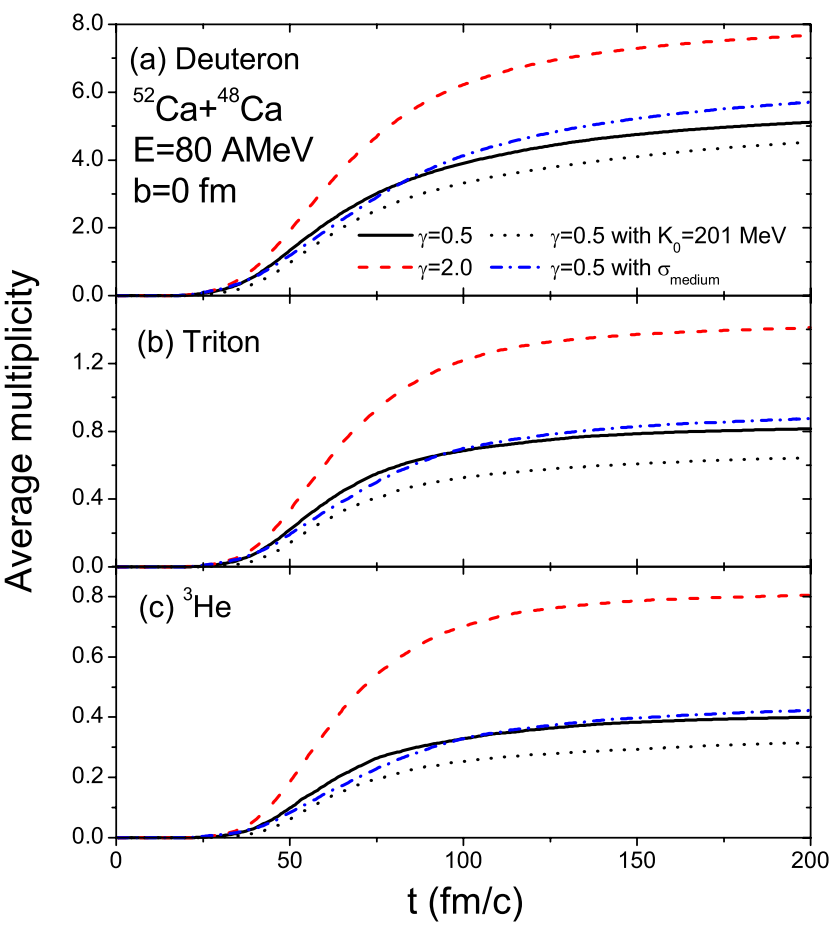

FIG. 1. (Color online) Time evolutions of the average multiplicity of (a) deuteron, (b) triton, and (c) ${ }^{3} \mathrm{He}$ from central collisions of ${ }^{52} \mathrm{Ca}+{ }^{48} \mathrm{Ca}$ at $E=80 \mathrm{MeV} /$ nucleon by using the soft (solid curves) and stiff (dashed curves) symmetry energies with a stiff nuclear compressibility $K_{0}=380 \mathrm{MeV}$ and free nucleon-nucleon cross sections. Results using the soft symmetry energy and free nucleonnucleon cross sections but $K_{0}=201 \mathrm{MeV}$ are shown by dotted curves, while those from the soft symmetry energy and $K_{0}$ $=380 \mathrm{MeV}$ but in-medium nucleon-nucleon cross sections are given by dash-dotted curves.

system is very sensitive to the density dependence of nuclear symmetry energy. Final multiplicities of deuteron, triton, and ${ }^{3} \mathrm{He}$ for the stiff symmetry energy are larger than those for the soft symmetry energy by $51 \%, 73 \%$, and $100 \%$, respectively. This is due to the fact that the stiff symmetry energy induces a stronger pressure in the reaction system and thus causes an earlier emission of neutrons and protons than in the case of the soft symmetry energy [37], leading to a stronger correlations among nucleons. Furthermore, the soft symmetry energy, which gives a more repulsive symmetry potential for neutrons and more attractive one for protons in low density region $\left(\leqslant \rho_{0}\right)$ than those from the stiff symmetry energy, generates a larger phase-space separation between neutrons and protons at freeze out, and thus a weaker correlations among nucleons. The larger sensitivity of the multiplicity of ${ }^{3} \mathrm{He}$ to the nuclear symmetry energy than those of triton and deuteron, as seen in Fig. 1, reflects the fact that the symmetry energy effect is stronger on lower momentum protons than neutrons [37].

Although the density dependence of nuclear symmetry energy affects appreciably the yield of light clusters, changing the incompressibility from $K_{0}=380$ to $201 \mathrm{MeV}$ (dotted curves) or using $\sigma_{\text {medium }}$ instead of $\sigma_{\text {exp }}$ (dash-dotted curves) only leads to a small change in the yield of these clusters, as shown in Fig. 1. This implies that the relative space-time 


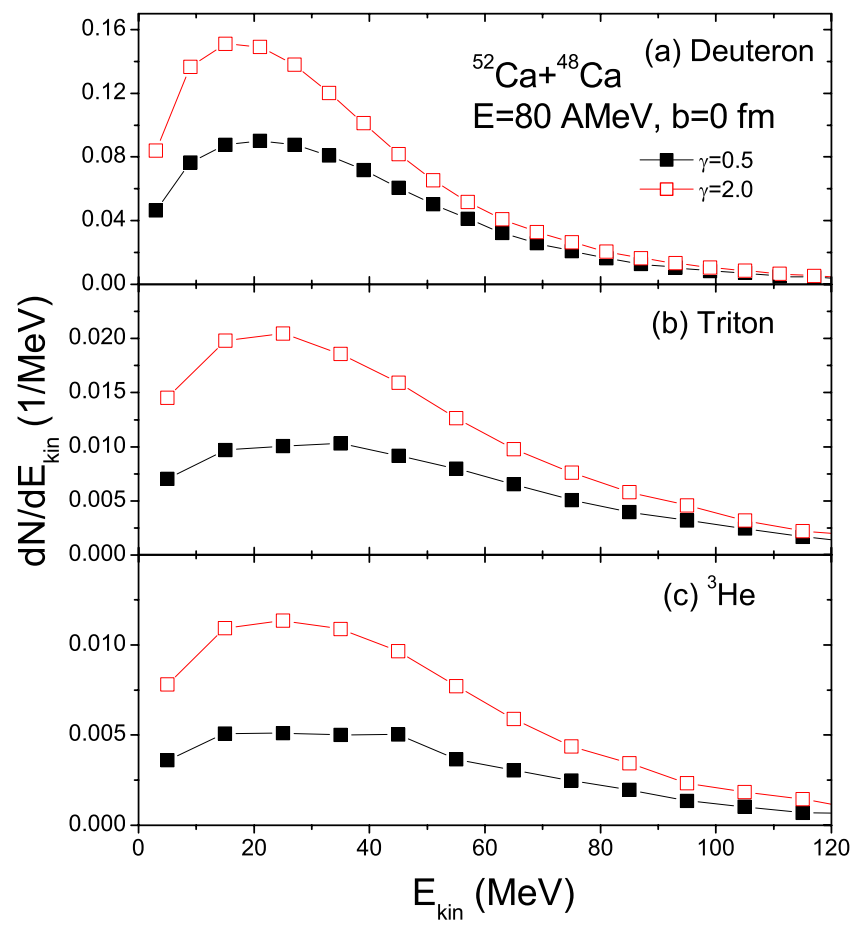

FIG. 2. (Color online) Kinetic energy spectra in the center-ofmass system for (a) deuteron, (b) triton, and (c) ${ }^{3} \mathrm{He}$ from central collisions of ${ }^{52} \mathrm{Ca}+{ }^{48} \mathrm{Ca}$ at $E=80 \mathrm{MeV} /$ nucleon by using the soft (solid squares) and stiff (open squares) symmetry energies with a stiff nuclear compressibility $K_{0}=380 \mathrm{MeV}$ and free nucleonnucleon cross sections.

structure of neutrons and protons at freeze out is not sensitive to the equation of state (EOS) of symmetric nuclear matter and in-medium nucleon-nucleon cross sections.

The kinetic energy spectra in the center-of-mass system for deuteron, triton, and ${ }^{3} \mathrm{He}$ are shown in Fig. 2 for both the soft (solid squares) and stiff (open squares) symmetry energies. It is seen that the symmetry energy has a stronger effect on the yield of low energy clusters than that of high energy ones. For example, the symmetry energy effect on the yield of deuteron, triton, and ${ }^{3} \mathrm{He}$ is about $60 \%, 100 \%$, and $120 \%$, respectively, if their kinetic energies are around $10 \mathrm{MeV}$, but is about $30 \%, 40 \%$, and $85 \%$, respectively, if their kinetic energies are around $100 \mathrm{MeV}$. This follows from the fact that lower energy clusters are emitted later in time when the size of nucleon emission source is relatively independent of nuclear symmetry energy, thus leading to a similar probability for nucleons to form light clusters. Since there are more low energy nucleons for a stiffer symmetry energy, more light clusters are thus produced. On the other hand, higher energy nucleons are emitted earlier when the size of emission source is more sensitive to the symmetry energy, with a smaller size for a stiffer symmetry energy. The probability for light cluster formation is thus larger for a stiffer symmetry energy. This effect is, however, reduced by the smaller number of high energy nucleons if the symmetry energy is stiffer. As a result, production of high energy light clusters is less sensitive to the stiffness of symmetry energy. This is different from that seen in the correlation functions between two nucleons with low relative momentum, where

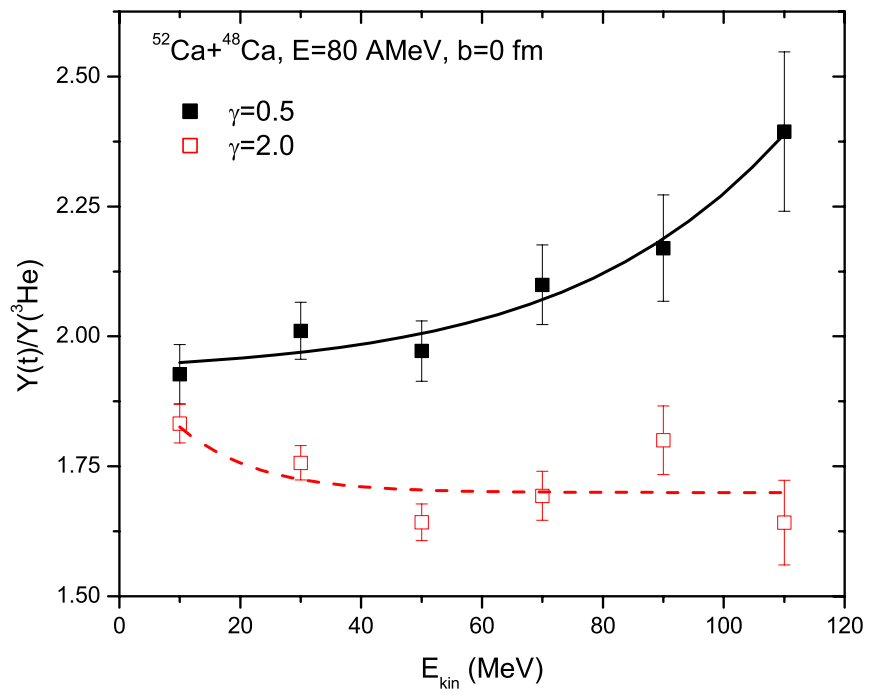

FIG. 3. (Color online) The ratio $t /{ }^{3} \mathrm{He}$ as a function of the cluster kinetic energy in the center-of-mass system by using the soft and stiff symmetry energies. The lines are drawn to guide the eye.

the symmetry energy effect is larger for nucleon pairs with higher kinetic energies [21], as they are only affected by the size of the emission source, not by the number of emitted nucleon pairs.

The isobaric yield ratio $t /{ }^{3} \mathrm{He}$ is less model-dependent and also less affected by other effects, such as the feedback from heavy fragment evaporation and the feed-down from produced excited triton and ${ }^{3} \mathrm{He}$ states. In Fig. 3, we show the $t /{ }^{3} \mathrm{He}$ ratio with statistical errors as a function of cluster kinetic energy in the center-of-mass system for the soft (solid squares) and stiff (open squares) symmetry energies. It is seen that the ratio $t /{ }^{3} \mathrm{He}$ obtained with different symmetry energies exhibits very different energy dependence. While the $t /^{3} \mathrm{He}$ ratio increases with kinetic energy for the soft symmetry energy, it decreases with kinetic energy for the stiff symmetry energy. The symmetry energy thus affects more strongly the ratio of high kinetic energy triton and ${ }^{3} \mathrm{He}$. For both soft and stiff symmetry energies, the ratio $t /{ }^{3} \mathrm{He}$ is larger than the neutron to proton ratio of the whole reaction system, i.e., $N / Z=1.5$. This is in agreement with results from both experiments and statistical model simulations for other reaction systems and incident energies [38-41]. It is interesting to note that although the yield of lower energy triton and ${ }^{3} \mathrm{He}$ is more sensitive to symmetry energy than higher energy ones, as shown in Fig. 2, their ratio at higher energy is affected more by the symmetry energy. Moreover, the energy dependence of the ratio $t /{ }^{3} \mathrm{He}$ is insensitive to the EOS of symmetric nuclear matter and in-medium nucleonnucleon cross sections. These features thus imply that the preequilibrium triton to ${ }^{3} \mathrm{He}$ ratio is also a sensitive probe to the density dependence of nuclear symmetry energy.

Isospin effects on cluster production and isotopic ratios in heavy ion collisions have been previously studied using either the lattice gas model [41] or a hybrid of IBUU and statistical fragmentation model [16]. These studies are, however, at lower energies than considered here, where effects due to multifragmentation as a result of possible gas-liquid 
phase transition may play an important role. Except deuterons, both tritons and ${ }^{3} \mathrm{He}$ are only about one per event in heavy ion collisions at $80 \mathrm{MeV} /$ nucleon. The number of other clusters, such as the alpha particle, is not large either $[42,43]$. In this case, the coalescence model is expected to be a reasonable model for determining the production of light clusters from heavy ion collisions. Furthermore, the effect obtained in present study will be enhanced if other clusters are emitted earlier, as the isospin asymmetry of the residue is increased.

In conclusion, using an isospin-dependent transport model together with a coalescence model for light cluster production, we have found that the nuclear symmetry energy affects significantly the production of light clusters in heavy-ion collisions induced by neutron-rich nuclei. More deuterons, tritons, and ${ }^{3} \mathrm{He}$ are produced with the stiff nuclear symmetry energy than the soft nuclear symmetry energy. This effect is particularly large when these clusters have lower kinetic en- ergies. Also, the isobaric ratio $t /{ }^{3} \mathrm{He}$, especially for higher energy tritons and ${ }^{3} \mathrm{He}$, shows a strong sensitivity to the density dependence of nuclear symmetry energy. It is further found that light cluster production is not sensitive to the isospin-independent part of nuclear equation of state and to the in-medium nucleon-nucleon cross sections. The study of light cluster production in heavy ion collisions induced by neutron-rich nuclei thus allows us to extract useful information about the density dependence of nuclear symmetry energy.

We thank Joe Natowitz for discussions on light clusters production in heavy ion collisions. This paper was based on the work supported by the U.S. National Science Foundation under Grant Nos. PHY-0098805 and PHY-0088934 as well as the Welch Foundation under Grant No. A-1358. L.W.C. was also supported by the National Natural Science Foundation of China under Grant No. 10105008.
[1] B.A. Li, C.M. Ko, and W. Bauer, Int. J. Mod. Phys. E 7, 147 (1998).

[2] W.D. Myers and W.J. Swiatecki, Nucl. Phys. A81, 1 (1966).

[3] K. Pomorski and J. Dudek, nucl-th/0205011.

[4] Isospin Physics in Heavy-Ion Collisions at Intermediate Energies, edited by Bao-An Li and W. Udo Schröder (Nova Science, New York, 2001).

[5] B.A. Brown, Phys. Rev. Lett. 85, 5296 (2000).

[6] K. Oyamatsu et al., Nucl. Phys. A634, 3 (1998).

[7] C.J. Horowitz and J. Piekarewicz, Phys. Rev. Lett. 86, 5647 (2001); Phys. Rev. C 63, 025501 (2001).

[8] R.J. Furnstahl, Nucl. Phys. A706, 85 (2002).

[9] H.A. Bethe, Rev. Mod. Phys. 62, 801 (1990).

[10] I. Bombaci, in, Isospin Physics in Heavy-Ion Collisions at Intermediate Energies (Ref. [4]), p. 35.

[11] J.M. Lattimer and M. Prakash, Astrophys. J. 550, 426 (2001).

[12] B.A. Li et al., Phys. Rev. Lett. 78, 1644 (1997).

[13] B.A. Li and C.M. Ko, Nucl. Phys. A618, 498 (1997).

[14] V. Baran et al., Nucl. Phys. A632, 287 (1998).

[15] H.S. Xu et al., Phys. Rev. Lett. 85, 716 (2000).

[16] W.P. Tan et al., Phys. Rev. C 64, 051901(R) (2001).

[17] M.B. Tsang et al., Phys. Rev. Lett. 86, 5023 (2001).

[18] B.A. Li et al., Phys. Rev. C 64, 054604 (2001).

[19] B.A. Li, Phys. Rev. Lett. 85, 4221 (2000).

[20] B.A. Li, Phys. Rev. Lett. 88, 192701 (2002); Nucl. Phys. A708, 365 (2002).

[21] L.W. Chen, V. Greco, C.M. Ko, and B.A. Li, Phys. Rev. Lett. 90, 162701 (2003)
[22] M. Gyulassy et al., Nucl. Phys. A402, 596 (1983).

[23] J. Aichelin et al., Phys. Rev. Lett. 58, 1926 (1987).

[24] V. Koch et al., Phys. Lett. B 241, 174 (1990).

[25] P. Pawlowski et al., Eur. Phys. J. A 9, 371 (2000).

[26] R. Mattiello et al., Phys. Rev. C 55, 1443 (1997).

[27] J.L. Nagle et al., Phys. Rev. C 53, 367 (1996).

[28] A. Polleri et al., Nucl. Phys. A661, 452c (1999).

[29] T. Ericsson and W. Weise, Pions and Nuclei (Clarendon, Oxford, 1988).

[30] C.R. Chen et al., Phys. Rev. C 33, 1740 (1986).

[31] B.A. Li et al., Phys. Rev. Lett. 76, 4492 (1996); B.A. Li and A.T. Sustich, ibid. 82, 5004 (1999).

[32] G.Q. Li and R. Machleidt, Phys. Rev. C 48, 1702 (1993); 49, 566 (1994)

[33] Q. Pan and P. Danielewicz, Phys. Rev. Lett. 70, 2062 (1993).

[34] J. Zhang et al., Phys. Rev. C 50, 1617 (1994).

[35] G.F. Bertsch and S. Das Gupta, Phys. Rep. 160, 189 (1988).

[36] H. Heiselberg and M. Hjorth-Jensen, Phys. Rep. 328, 237 (2000).

[37] L.W. Chen, V. Greco, C.M. Ko, and Bao-An Li, Phys. Rev. C (in press)

[38] J. Cibor et al., Phys. Lett. B 473, 29 (2000).

[39] K. Hagel et al., Phys. Rev. C 62, 034607 (2000).

[40] M. Veselky et al., Phys. Lett. B 497, 1 (2001).

[41] Ph. Chomaz and F. Gulminelli, Phys. Lett. B 447, 221 (1999).

[42] B.V. Jacak et al., Phys. Rev. C 35, 1751 (1987).

[43] B. Borderie et al., Phys. Lett. B 388, 224 (1996). 